\title{
Structure Function Resummation in small-x QCD
}

\author{
Guido Altarelli* \\ Dipartimento di Fisica "E.Amaldi" and Sezione INFN, Università Roma Tre, Roma, Italy \\ and CERN, Department of Physics, Theory Division, Genève, Switzerland \\ E-mail: guido.altarelli@cern.ch
}

\section{Richard D. Ball}

School of Physics, University of Edinburgh, Edinburgh EH9 3JZ, Scotland and CERN, Department of Physics, Theory Division, Genève, Switzerland

E-mail: rdb@ph.ed.ac.uk

\section{Stefano Forte}

Dipartimento di Fisica e Sezione INFN, Università di Milano, Milan, Italy

E-mail: stefano.forte@mi.infn.it

\begin{abstract}
We summarize our recent results on small $x$ resummation in full QCD with $n_{f}$ quark flavours and discuss their phenomenological impact in the extraction of parton distributions from present day structure function data and their extrapolation to the kinematics relevant for future colliders such as the LHC.
\end{abstract}

8th International Symposium on Radiative Corrections (RADCOR)

October 1-5 2007

Florence, Italy

\footnotetext{
${ }^{*}$ Speaker.
} 
Higher order calculations in perturbative QCD have progressed in an extraordinary way in recent years, motivated by the needs of accurate phenomenology at the LHC. The frontier of present-day perturbative calculations is the next-to-next-to leading (NNLO) order [1], due to the availability of a variety of novel computational techniques. Results for cross-sections at NNLO can be used thanks to the recent determination [2] of three-loop splitting functions which drive NNLO perturbative evolution. However, perturbative evolution at NNLO is unstable in the high energy ( $\operatorname{small} x$ limit): the size of the NNLO corrections diverges as $x \rightarrow 0$ at fixed scale.

Small $x$ resummation, which should take care of this instability, has a rather long history, starting with the original determination of leading high energy corrections [3] and their inclusion in perturbative anomalous dimensions [4]. Until quite recently, however, its relevance for phenomenology has been modest: since the advent of HERA data, it is clear that a NLO description of observed scaling violation is perfectly adequate [5, 6], and the data show no evidence for large small $x$ effects. The reason why nominally large corrections seem to have no impact has been obscure for a long time. However, due to some accidental zeros in coefficients, small $x$ contributions to NLO perturbative evolution are small, so in practice one could simply ignore the issue for all practical purposes. At NNLO, however, small $x$ terms are large, the perturbative instability is manifest, and resummation becomes mandatory.

Fortunately, over the last few years a fully resummed approach to perturbative evolution has been constructed. Within this approach, it is possible to understand why fixed perturbative order corrections are very large at small $x$, yet their full resummation leads to a considerable softening of small $x$ terms, consistent with the fact that the HERA data do not show any large departure from NLO predictions. In order to obtain stable resummed results one must satisfy various physical constraints, such as momentum conservation, renormalization group invariance and gluon exchange symmetry. These require the inclusion of several classes of terms which are formally subleading in comparison to the series of leading or next-to-leading small $x$ logs. Once these constraints are enforced, the resummed perturbative expansion at small $x$ becomes stable, and one no longer sees the strong small $x$ enhancement or suppression that the leading [3] and subleading [7]-[10] small $x$ logs would give.

A comparison of existing approaches to this resummation, as discussed respectively in refs. [11, 12, 13] (ABF approach) and [14, 15] (CCSS approach), shows [6] that they yield results which agree with each other within the expected theoretical uncertainty. They agree in including the physical assumptions listed above. They differ mostly because the CCSS approach is built up within the BFKL framework, by improving the BFKL kernel through the inclusion of terms which become important in the collinear region, while the $\mathrm{ABF}$ approach is based on the construction of an improvement of the GLAP anomalous dimension through the inclusion of terms that become important in the small $x$ region. The fact that they lead to very similar results is thus a consequence of the "duality" which relates the BFKL and GLAP description of perturbative evolution: at leading twist, they can describe the same physics, provided the respective evolution kernels are suitably matched $[13,17,18]^{1}$.

Because the leading high energy corrections are dominated by gluon exchange, the resumma-

\footnotetext{
${ }^{1}$ Small- $x$ corrections to polarized structure functions, of different form and origin than those considered here, have been recently discussed in ref. [16]
} 

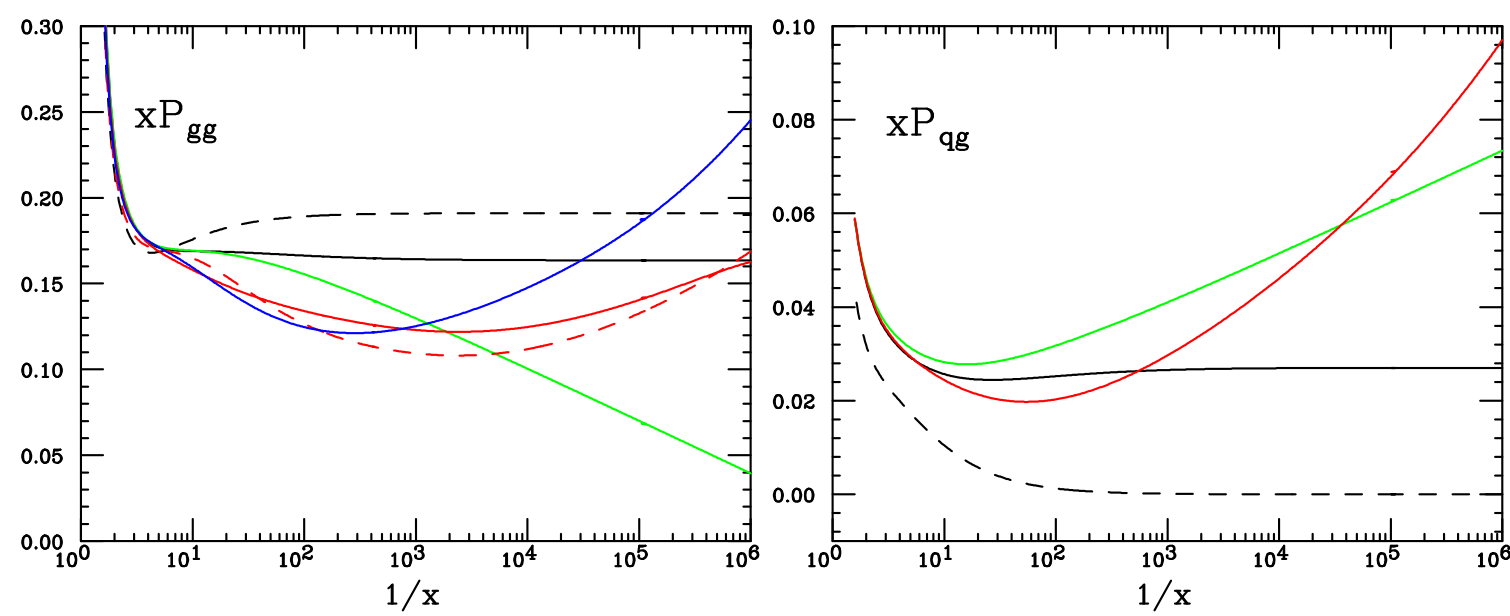

Figure 1: The splitting functions $x P_{g g}$ and $x P_{q g}$ for $n_{f}=4$ and $\alpha_{s}=0.2$ as a function of $x$. Fixed order perturbation theory LO (black, dashed); NLO (black, solid) and NNLO (green, solid) and resummed LO (red, dashed) and NLO in $\mathrm{Q}_{0} \overline{\mathrm{MS}}$ scheme (red, solid) and in the $\overline{\mathrm{MS}}$ scheme (blue, solid).

tion is most easily performed in the pure Yang-Mills theory, and indeed the full resummed results for perturbative evolution of refs. [11,15] have been obtained with $n_{f}=0$. In order to actually get predictions for (flavour singlet) physical observables, one needs the full two-by-two matrix of splitting functions, and the set of hard coefficient functions for the desired observables, in a given factorization scheme. The construction of resummed splitting functions is simplified by the fact that only one of the two eigenvalues of the singlet anomalous dimension matrix has leading $N=0$ singularities: hence, only this eigenvalue is affected by the resummation. In deep-inelastic scattering, the construction of resummed coefficient functions is further simplified by the fact that virtual photons at leading order only couple to quarks. This implies that there always exist schemes where only one parton (quark or gluon) contributes to each of the structure functions $F_{2}$ and $F_{L}$. It follows that in any factorization scheme the resummation of the coefficient is specified in terms of a single function for each structure function (these functions have been determined in ref. [19], where they are called $h_{2}$ and $h_{L}$ for $F_{2}$ and $F_{L}$ respectively.)

Hence, (at least) two strategies are available for the construction of resummed observables in deep-inelastic scattering. The first possibility is to simply pick a factorization scheme, then construct resummed two-by-two evolution kernels and resummed coefficient functions in that scheme. This program was started in ref. [20], where the full $n_{f} \neq 0$ resummed evolution matrix was constructed by extending a BFKL-like approach to coupled quark and gluon evolution, along the lines of the approach of refs. $[14,15]$. This has the advantage of giving evolution equations for off-shell, unintegrated parton distributions, but it has the shortcoming of providing results in a factorization scheme which only coincides with $\overline{\mathrm{MS}}$ up to the next-to-leading fixed order, and differs from it at the resummed level. Available resummed coefficient functions [19], which are given in $\overline{\mathrm{MS}}$ or DIS, are not readily combined with the evolution kernels determined in this way. Similar problems were encountered in ref. [21], where resummed structure functions were obtained by combining resummed anomalous dimensions and coefficients determined in different factorizaton schemes.

A second possibility consists of taking advantage of the peculiar fact that both the resumma- 

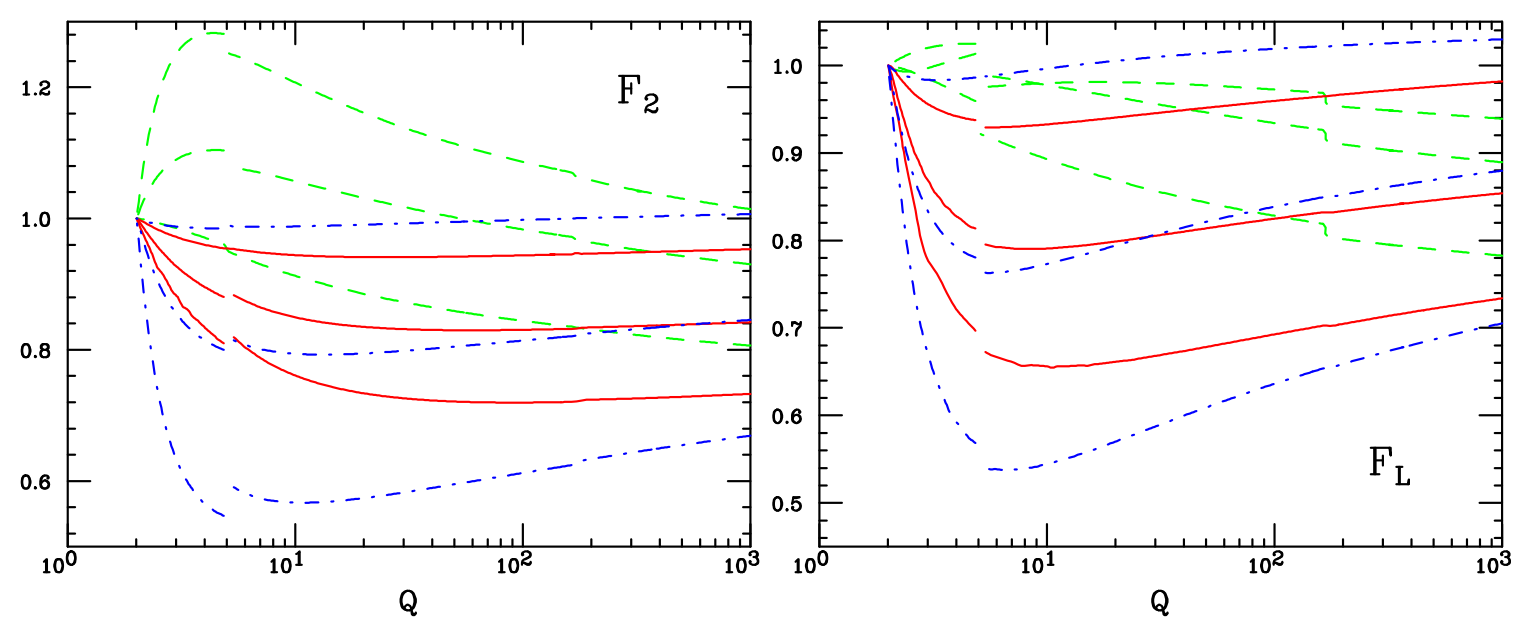

Figure 2: The $K$-factors singlet $F_{2}$ and $F_{L}$ structure functions at fixed $x=10^{-2}, 10^{-4}$ or $10^{-6}$ as a function of $Q$ with $\alpha_{s}$ running and $n_{f}$ varied (the breaks in the curves correspond to the $\mathrm{b}$ and t quark thresholds). Fixed order perturbation theory NNLO (green, dashed); resummed NLO in $\mathrm{Q}_{0} \overline{\mathrm{MS}}$ scheme (red, solid), resummed NLO in the $\overline{\mathrm{MS}}$ scheme (blue, dot-dashed).

tion of the anomalous dimensions and that of the deep-inelastic coefficients for a given structure function are each determined in terms of a single function: it is only when specifying the factorization scheme that a matrix of anomalous dimensions and a vector of coefficients for each structure function are obtained. It follows that if one has full control of the transformation between factorization schemes at the resummed level, one can obtain results for resummed coefficient functions and anomalous dimensions in any scheme.

This approach was pursued in ref. [22], where by making use of the general techniques for cross-section resummation developed in ref. [23] we were able to compute the full $n_{f} \neq 0$ matrix of resummed singlet splitting functions (see fig. 1) together with the coefficients $C_{q}^{i}$ and $C_{g}^{i}(\mathrm{i}=2, \mathrm{~L})$ for the singlet structure functions $F_{2}$ and $F_{L}$, respectively, in various commonly used schemes such as the $\overline{\mathrm{MS}}$ scheme. This then allowed us to determine for the first time the structure functions at a fully resummed level in a consistent factorization scheme. While referring to ref. [22] for a full discussion of the resummed splitting functions and coefficient functions, we focus here on the impact of the resummation on physical observables, and on the extraction of parton distributions from them.

Thus, in fig. 2 we compare the effects of resummed evolution with those of fixed order perturbative evolution at NLO (which is the usual baseline for most current fits) and also at NNLO. We show the $K$-factor (defined as resummed/(NLO fixed order pert)) for the singlet $F_{2}$ structure function at fixed values of $x$ as a function of $Q$. For each $x$ value we present three curves: the resummed case in the $\mathrm{Q}_{0} \overline{\mathrm{MS}}$ scheme, the corresponding plot in the $\overline{\mathrm{MS}}$ scheme, and the NNLO fixed order perturbative. The starting point at $Q_{0}=2$ is taken to be the same for the structure functions plotted in the three curves. In the expanded linear scale of these plots we can see the moderate scheme difference between the $\mathrm{Q}_{0} \overline{\mathrm{MS}}$ and the $\overline{\mathrm{MS}}$ schemes, which is left after combining coefficients and parton densities, which, individually, show a much more pronounced scheme dependence.

For $F_{2}$ the effect of resummation, at sufficiently small $x$ values, goes in the opposite direction 

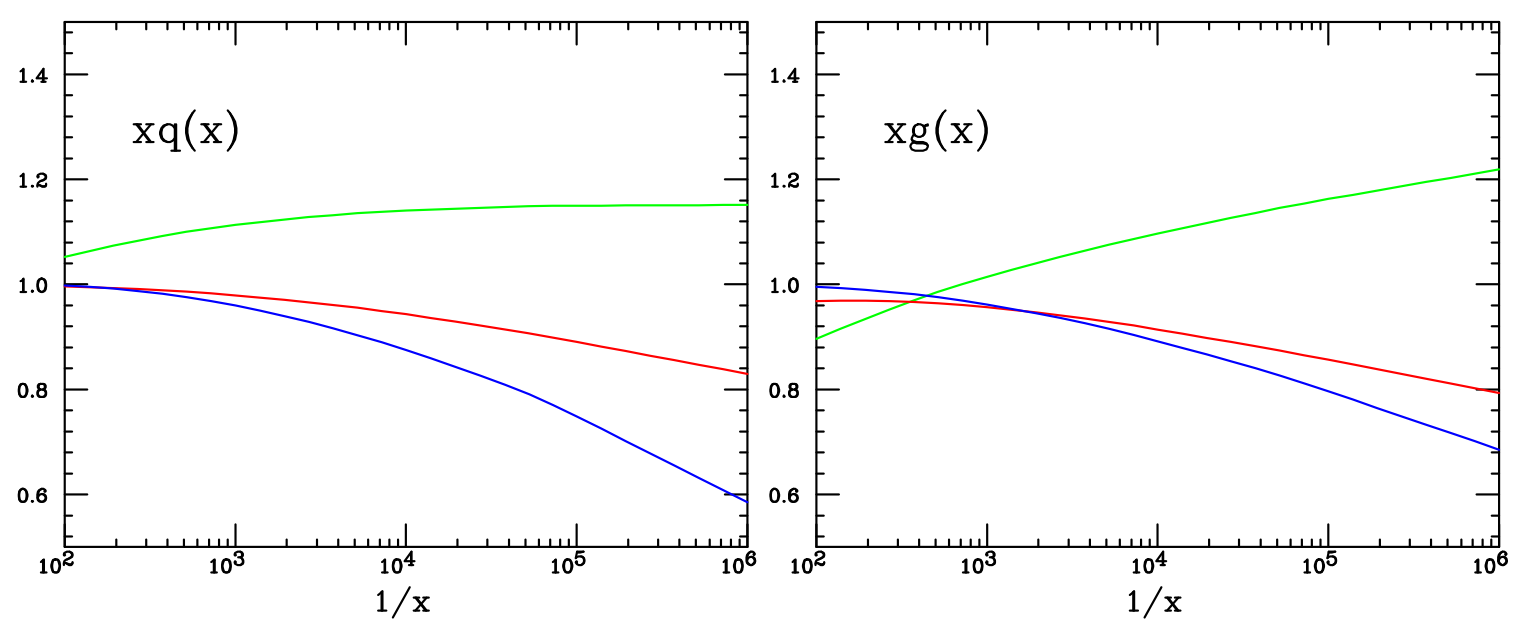

Figure 3: The $K$-factors, defined as the ratio of the fixed order NNLO or resummed to the NLO fixed order result, for $x q$ and $x g$ when $F_{2}$ and $F_{L}$ are fixed at the reference scale $Q_{0}=5 \mathrm{GeV}$. Results are shown at $Q=Q_{0}$ as as function of $x$ in the range $x=10^{-2}$ or $10^{-6}$. The curves (top to bottom at small $x$ ) are: fixed order perturbation theory NNLO (green); resummed NLO in $\mathrm{Q}_{0} \overline{\mathrm{MS}}$ scheme (red), resummed NLO in the $\overline{\mathrm{MS}}$ scheme (blue).

to the NNLO perturbative evolution: the resummed $K$-factor is less than one, corresponding to a smaller structure function at higher scales than with fixed order perturbative NLO evolution. The effect of the resummation is somewhat larger than that of the NNLO. This shows that for $x \lessgtr 0.2$ the inclusion of unresummed NNLO terms is actually counterproductive.

In view of using parton distributions extracted from HERA data for physics at the LHC, it is also interesting to study how the quark and gluon distributions change when the resummation is switched on, while imposing that the measurable structure functions be unchanged. We do this in fig. 3, where we display the $K$-factors for the input quark and gluon distributions as a function of $x$, with the input scale $Q_{0}=5 \mathrm{GeV}$. Here the structure functions $F_{2}$ and $F_{L}$ at the input scale are kept fixed, but the coefficient functions are changed depending on the perturbative approximation employed. Whereas at NNLO the quark and gluon distributions are enhanced with respect to the $\mathrm{NLO}$, at the resummed level they are suppressed; the suppression becomes increasingly significant at smaller $x$. The effect on the parton distribution is sizable, but when evolving up the scale dependence tends to reduce this effect leading to the more moderate corrections displayed in figure 2 . The general conclusion, however, is that if resummation effects are disregarded, the associated error in extracting parton distributions at HERA and evolving them up at LHC is of order of about $5 \%$ at $x \sim 10^{-3}$, and as large as $20 \%$ for low values of $x \sim 10^{-6}$.

\section{Acknowledgements}

Two of us, G.A. and S.F., acknowledge that the present work has been supported in part by the Italian Ministero dell'Universita' e della Ricerca Scientifica, under the PRIN program for 2007-08. The work of R.D.B. has been done in the context of the Scottish Universities' Physics Alliance. This work was partly supported by the Marie Curie Research and Training network HEPTOOLS under contract MRTN-CT-2006-035505. 


\section{References}

[1] T. Gehrmann, arXiv:0709.0351 [hep-ph].

[2] S. Moch, J. A. M. Vermaseren and A. Vogt, Nucl. Phys. B691 (2004) 129; A. Vogt, S. Moch and J. A. M. Vermaseren, Nucl. Phys. B688 (2004) 101.

[3] L.N. Lipatov, Sov. Jour. Nucl. Phys. 23 (1976) 338; V.S. Fadin, E.A. Kuraev and L.N. Lipatov, Phys. Lett. 60B (1975) 50; Sov. Phys. JETP 44 (1976) 443; 45 (1977) 199; Y.Y. Balitski and L.N.Lipatov, Sov. Jour. Nucl. Phys. 28 (1978) 822.

[4] T. Jaroszewicz, Phys. Lett. B116 (1982) 291.

[5] R. D. Ball and S. Forte, Phys. Lett. B335 (1994) 77; $\mathbf{B 3 3 6}$ (1994) 77.

[6] M. Dittmar et al., arXiv:hep-ph/0511119.

[7] V. S. Fadin and L. N. Lipatov, Phys. Lett. B429 (1998) 127.

[8] V.S. Fadin et al, Phys. Lett. B359 (1995) 181; Phys. Lett. B387 (1996) 593; Nucl. Phys. B406 (1993) 259; Phys. Rev. D50 (1994) 5893; Phys. Lett. B389 (1996) 737; Nucl. Phys. B477 (1996) 767;

Phys. Lett. B415 (1997) 97; Phys. Lett. B422 (1998) 287.

[9] G. Camici and M. Ciafaloni, Phys. Lett. B412 (1997) 396; Phys. Lett. B430 (1998) 349.

[10] V. del Duca, Phys. Rev. D54 (1996) 989; Phys. Rev. D54 (1996) 4474

V. del Duca and C.R. Schmidt, Phys. Rev. D57 (1998) 4069

Z. Bern, V. del Duca and C.R. Schmidt, Phys. Lett. B445 (1998) 168.

[11] R. D. Ball and S. Forte, Phys. Lett. B465 (1999) 271.

[12] G. Altarelli, R. D. Ball and S. Forte, Nucl. Phys. B575, 313 (2000); hep-ph / 0001157 ; Nucl. Phys. B599 (2001) 383; hep-ph / 0104246.

[13] G. Altarelli, R. D. Ball and S. Forte, Nucl. Phys. B621 (2002) 359; Nucl. Phys. B674 (2003) 459; hep-ph/0310016; Nucl. Phys. B742 (2006) 1; hep-ph/0606323.

[14] G. Salam, Jour. High Energy Phys. 9807 (1998) 19.

[15] M. Ciafaloni and D. Colferai, Phys. Lett. B452 (1999) 372;

M. Ciafaloni, D. Colferai and G. P. Salam, JHEP0007 (2000) 054; Phys. Rev. D60 (1999) 114036; M. Ciafaloni, D. Colferai, G. P. Salam and A. M. Stasto, Phys. Rev. D66 (2002) 054014; Phys. Lett. B 576 (2003) 143; Phys. Rev. D68 (2003) 114003; Phys. Lett. B587 (2004) 87; see also G. P. Salam, hep-ph/0501097.

[16] B.I. Ermolaev, M. Greco and S.I. Troyan. Eur. Phys. J C50 (2007) 823,859.

[17] R. D. Ball and S. Forte, Phys. Lett. B405 (1997) 317.

[18] R. D. Ball and S. Forte, Nucl. Phys. B742 (2006) 158.

[19] S. Catani and F. Hautmann, Phys. Lett. B315 (1993) 157; Nucl. Phys. B427 (1994) 475.

[20] M. Ciafaloni, D. Colferai, G. P. Salam and A. M. Stasto, arXiv:0707.1453 [hep-ph]. See also M. Ciafaloni, these proceedings.

[21] C. D. White and R. S. Thorne, Phys. Rev. D75 (2007) 034005 [arXiv:hep-ph/0611204].

[22] G. Altarelli, R. D. Ball and S. Forte, arXiv:0802.0032 [hep-ph].

[23] R. D. Ball, arXiv:0708.1277 [hep-ph]. 